\title{
From Pappus Theorem to parameter spaces of some extremal line point configurations and applications
}

\author{
Magdalena Lampa-Baczyńska $^{1}$ • Justyna Szpond ${ }^{1}$ (D)
}

Received: 15 May 2016 / Accepted: 4 November 2016 / Published online: 14 November 2016

(C) The Author(s) 2016. This article is published with open access at Springerlink.com

\begin{abstract}
In the present work we study parameter spaces of two line point configurations introduced by Böröczky. These configurations are extremal from the point of view of the Dirac-Motzkin Conjecture settled recently by Green and Tao (Discrete Comput Geom 50:409-468, 2013). They have appeared also recently in commutative algebra in connection with the containment problem for symbolic and ordinary powers of homogeneous ideals (Dumnicki et al. in J Algebra 393:24-29, 2013) and in algebraic geometry in considerations revolving around the Bounded Negativity Conjecture (Bauer et al. in Duke Math J 162:1877$1894,2013)$. We show that the parameter space of what we call $\mathbb{B} 12$ configurations is a three dimensional rational variety. As a consequence we derive the existence of a three dimensional family of rational $\mathbb{B} 12$ configurations. On the other hand the parameter space of $\mathbb{B} 15$ configurations is shown to be an elliptic curve with only finitely many rational points, all corresponding to degenerate configurations. Thus, somewhat surprisingly, we conclude that there are no rational $\mathbb{B} 15$ configurations.
\end{abstract}

Keywords Arrangements of lines · Combinatorial arrangements · Containment problem for powers of ideals · Pappus Theorem

Mathematics Subject Classification $14 \mathrm{~N} 20 \cdot 52 \mathrm{C} 35 \cdot 32 \mathrm{~S} 22 \cdot 52 \mathrm{C} 30 \cdot 13 \mathrm{~F} 20$

Justyna Szpond was partially supported by National Science Centre, Poland, Grant 2014/15/B/ST1/02197.

Justyna Szpond
szpond@gmail.com

Magdalena Lampa-Baczyńska

lampa.baczynska@wp.pl

1 Department of Mathematics, Pedagogical University of Cracow, Podchorążych 2, 30-084 Kraków, Poland 


\section{Introduction}

Line point configurations are a classical subject of study in various branches of mathematics, e.g. geometry, combinatorics, topology. Recently they have appeared in commutative algebra in connection with the Containment Problem between symbolic and ordinary powers of ideals $[10,17]$ and in algebraic geometry in connection with the Bounded Negativity Conjecture, more precisely in the computations of the linear Harbourne constant $[2,9,18]$. In both of these problems the field of definition of the studied configurations plays an important role. In the present note we focus on two types of real (i.e. defined over $\mathbb{R}$ ) configurations of lines. More specifically, configurations studied here were introduced by Böröczky, see [5] for their probably first appearance in print. It has been realized that these configurations are relevant from the point of view of two problems in combinatorial geometry: the number of triangles in the simplicial partition of the plane by lines and in the Dirac-Motzkin Conjecture, see $[11,12]$. Recall that this Conjecture predicts a lower bound on the number of ordinary intersection points (i.e. points where only two lines intersect) of a configuration of real lines, which do not form a pencil. Böröczky examples are extremal from the point of view of this Conjecture as the number of ordinary intersection points they generate is as low as possible, cf. [12, Theorem 1.2]. We denote a Böröczky configuration of $d$ lines by $\mathbb{B} d$. The original configuration construction uses trigonometric functions. In this note we will give an elementary, geometric construction of the configuration $\mathbb{B} 12$ based just on the Pappus Theorem. This construction combined with an algebraic method of coordinatization introduced by Sturmfels [16] allows us to study the parameter space of $\mathbb{B} 12$ configurations. This parameter space is a quasi-projective rational variety (Theorem A). We describe its compactification and discuss briefly resulting degenerate configurations in the Appendix. Next we pass to $\mathbb{B} 15$ configurations and show that somewhat surprisingly their compactified parameter space is an elliptic curve with very few rational points (Theorem B).

Our interest in these configurations has been motivated by the following path of research. The Containment Problem for ordinary and symbolic powers of ideals in its simplest form is the following question raised by Huneke around 2000.

Question 1 (Huneke) Let $\mathbb{K}$ be an arbitrary field and let $I$ be an ideal of finitely many points in $\mathbb{P}^{2}(\mathbb{K})$. Is then the third symbolic power of $I$ a subset of the second ordinary power of $I$ ? That is does the containment

$$
I^{(3)} \subset I^{2}
$$

hold?

In 2013 Dumnicki, Szemberg and Tutaj-Gasińska discovered a first counterexample to the containment in (1), see [10]. They showed that for $\mathbb{K}=\mathbb{C}$ the containment in (1) fails for the ideal of all intersection points of a certain configuration of complex lines (the so called dual Hesse configuration). In [6] it has been noticed that the ideal of all non ordinary intersection points of a $\mathbb{B} d$ configuration with $d \geq 12$, provides a counterexample to (1) for $\mathbb{K}=\mathbb{R}$. Harbourne and Seceleanu observed in passing in [13] that a $\mathbb{B} 12$ configuration can be defined over the rational numbers and suggested that all Böröczky configurations can be defined over $\mathbb{Q}$. We show that at least for $\mathbb{B} 15$ this is not the case (Corollary 1 ) and this leads naturally to an open Problem 1.

Our main results are

Theorem A All $\mathbb{B} 12$ configurations form a 3 dimensional family parameterized by a rational variety (an open set in $\left.\left(\mathbb{P}^{1}(\mathbb{R})\right)^{3}\right)$. 
Theorem B All $\mathbb{B} 15$ configurations form a 1 dimensional family parameterized by an elliptic curve.

Remark 1 It would be desirable to know parameter spaces for all $\mathbb{B} d$ configuration, where $d$ is a multiple of 3 . Preliminary considerations indicate that they are all of general type. We hope to come back to this in the next future.

Theorem B has the following, somewhat surprising, consequence.

Corollary 1 There is no $\mathbb{B} 15$ configuration over the rational numbers.

As a consequence we conclude that from the point of view of combinatorics there is so far just one known rational counterexample to Huneke's Question 1. This is quite striking and suggests for further considerations the following problem.

Problem 1 Construct more rational counterexamples to the containment problem.

More generally, it is natural to wonder if configurations of rational lines enjoy some special combinatorial properties. We think here about analogies of the Green-Tao Theorem which asserts for real configurations of lines the existence of high enough number of ordinary intersection points, which is, for example, not the case for configurations of complex lines. One has to gather more evidence in order to formulate even a conjectural answer to the following question.

Problem 2 Provide combinatorial constrains for configurations of rational lines.

\section{A configuration of 12 lines with 19 triple points}

\subsection{Geometric construction}

In this section we provide a direct geometric construction of the Böröczky configuration of 12 lines, which works in a projective plane defined over an arbitrary field with sufficiently many elements.

The main auxiliary results which come into the argument are the following.

Theorem 1 (Pappus's Theorem) If triangles $A B C$ and $D E F$ are perspective in two ways with perspective centers $P, Q$, then there is also a third perspective center $R$ (see Fig. 1).

The following version of Bezout's Theorem is taken from [1].

Theorem 2 If two projective curves $C$ and $D$ in $\mathbb{P}^{2}$ of degree $n$ intersect at exactly $n^{2}$ points and if $n \cdot m$ of these points lie on irreducible curve $E$ of degree $m<n$, then the remaining $n \cdot(n-m)$ points lie on curve of degree at most $n-m$.

Now we are in the position to run our geometric construction.

To begin with let $A, B$ and $C$ be non-collinear points in $\mathbb{P}^{2}$. Then choose three points $D, E, F$ on the lines $A C, A B$ and $B C$, respectively, different from the points $A, B, C$. The points $D, E, F$ will be parameters of our construction. Then we define consecutively the following 6 points. 
Fig. 1 Pappus's Theorem

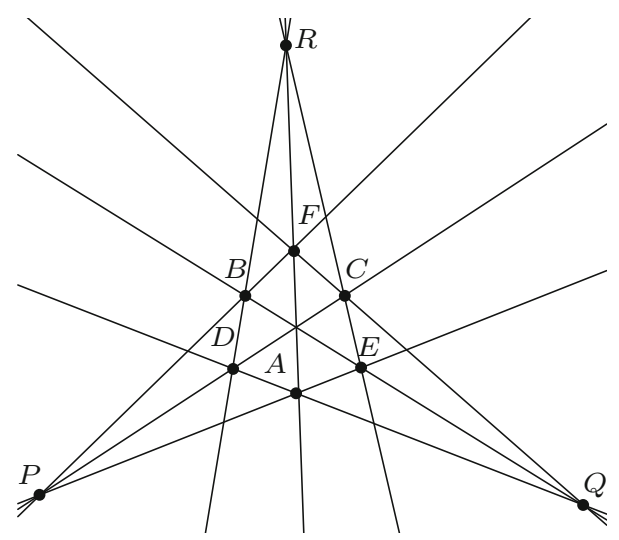

$$
\begin{gathered}
G=A F \cap B D, \\
H=B D \cap E F, \\
I=B C \cap E D, \\
J=A F \cap E D, \\
K=A C \cap E F, \\
L=H J \cap I K .
\end{gathered}
$$

At this stage an additional incidence comes into the picture.

Lemma 1 The line AB passes through the point $L$.

Proof The incidences coming from the construction so far are indicated in Fig. 2.

Since triangles $B J K$ and $A H I$ are perspective with perspective centers $D, F$, Theorem 1 yields that there is the third perspective center $L=H J \cap I K \cap A B$. In particular, the points $A, B$ and $L$ are collinear.

Then we define the remaining 7 points:

$$
\begin{aligned}
& M=B D \cap I K, \\
& N=A C \cap H J, \\
& O=H J \cap B C, \\
& P=A F \cap I K, \\
& Q=E F \cap N G, \\
& R=D E \cap C P, \\
& S=C P \cap N G .
\end{aligned}
$$

Here we claim two additional collinearities.

Lemma 2 From the above assumptions it follows that the points $A, B$ and $S$ are collinear.

Proof In order to prove the collinearity of points $A, B$ and $S$ we introduce an extra point $T=F N \cap D P$.

Claim The points $T, L$ and $E$ are collinear. 


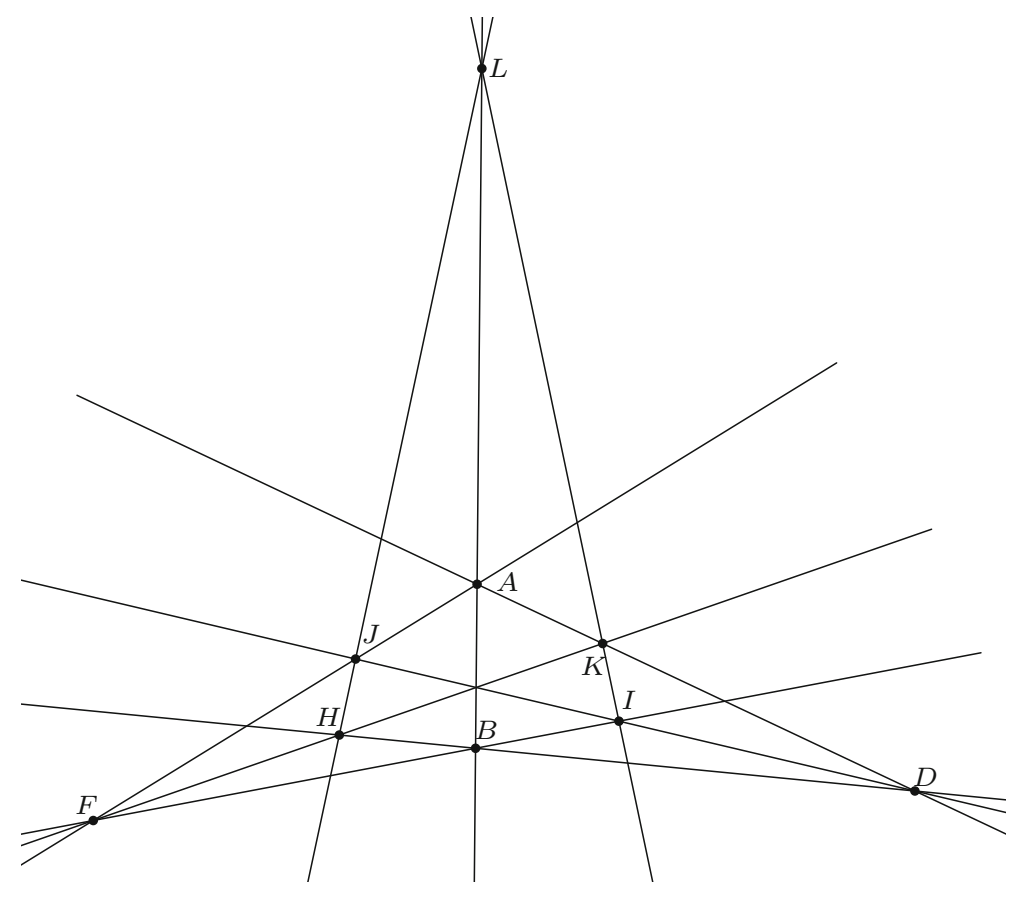

Fig. $2 \mathbb{B} 12$ construction - phase 1

Note that this implies the collinearity of $T$ with $A$ and $B$ as well. Figure 3 contains points relevant for the proof of the Claim.

Pappus Theorem applied to triangles $N P E$ and $T J K$ yields that points $L, T$ and $E$ are collinear.

By the same token we show the collinearity of points $T, S$ and $B$. All relevant points are marked on Fig. 4. We leave the exact argument to the reader.

We conclude that the points $A, B$ and $S$ are collinear as asserted in the Lemma.

Lemma 3 In the construction above the points $M, O, Q$ and $R$ are collinear.

Proof In order to prove the collinearity of points $O, Q, R$ we take two reducible curves $\alpha=K N \cup F G \cup B L$ and $\beta=K L \cup G N \cup B F$ of degree 3 . They intersect at 9 points and since points $C, P, S$ lie on a line, Theorem 2 yields that the remaining 6 points: $B, F, G, K$, $L, N$ lie on a conic, see Fig. 5 .

Now we will show that the points $M, O$ and $Q$ lie on one line. In order to prove it we take the reducible cubics $\alpha=K L \cup B F \cup G N$ and $\beta=B G \cup L N \cup F K$. They intersect in 9 points and from the above statement we know that points $B, F, G, K, L, N$ lie on a conic, hence the remaining three points $M, O$ and $Q$ are collinear by Theorem 2, see Fig. 6 .

Analogously, using Theorem 2 and taking curves $\alpha^{\prime}=B D \cup C P \cup J L$ and $\beta^{\prime}=$ $B L \cup C D \cup J P$ one can prove that the points $B, C, D, J, L$ and $P$ lie on a conic, and finally taking $\alpha^{\prime \prime}=B D \cup C P \cup J L$ and $\beta^{\prime \prime}=B C \cup D J \cup L P$ one can prove that the points $M$, $O$ and $R$ are collinear.

The collinearities of $M, O, Q$ and $M, O, R$ imply that the points $M, O, Q$ and $R$ lie on a line as asserted.

The resulting configuration is indicated in Fig. 7. 


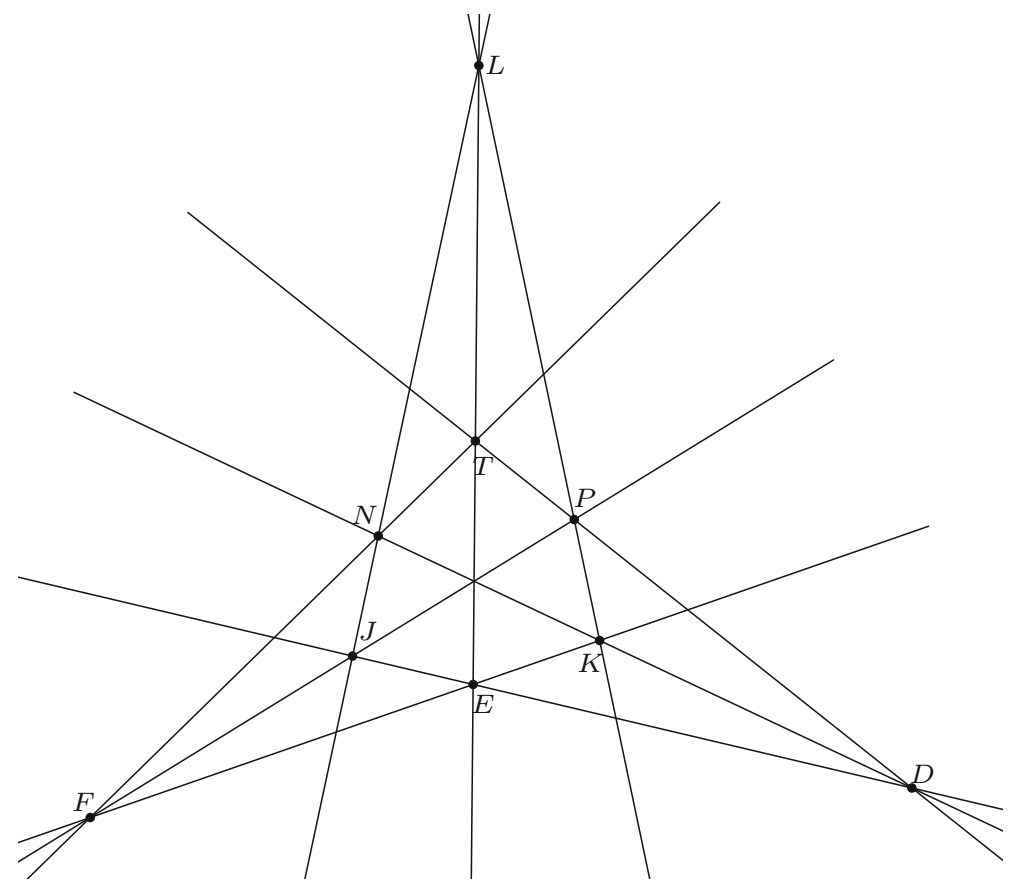

Fig. $3 \mathbb{B} 12$ construction - phase 2

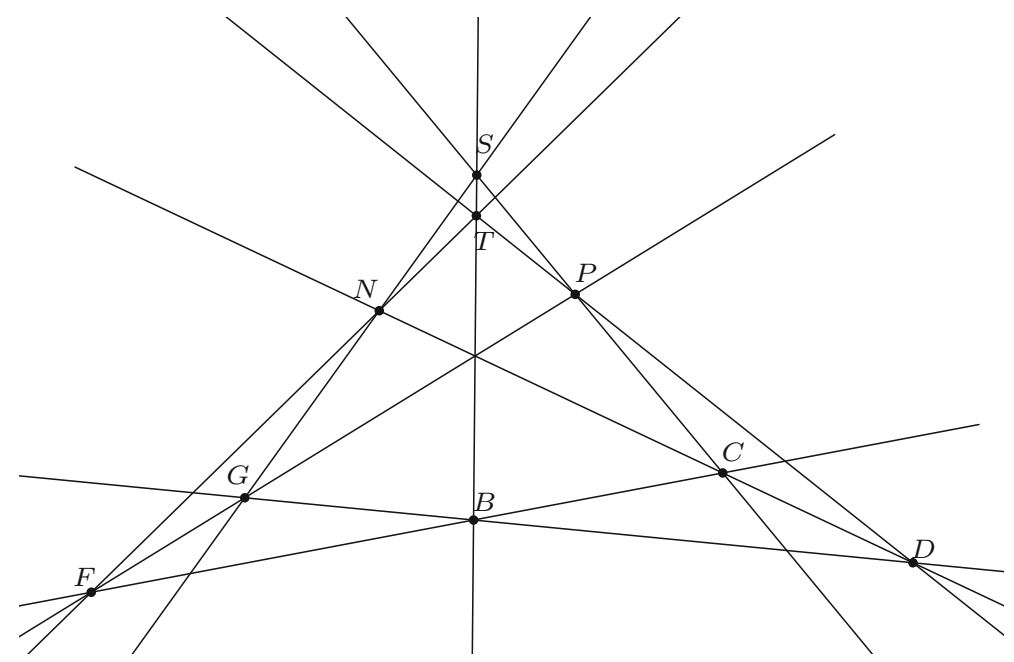

Fig. $4 \mathbb{B} 12$ construction - phase 3

\subsection{Algebraic proof}

In this section we provide another construction using algebraic methods in the spirit of Sturmfels' [16] and Tao's survey [19]. This approach makes the study of the parameter space, in particular degenerate cases, accessible. Since any two lines on the projective plane are projectively equivalent we may assume that points $A, B$ and $C$ are fundamental points, i.e. 


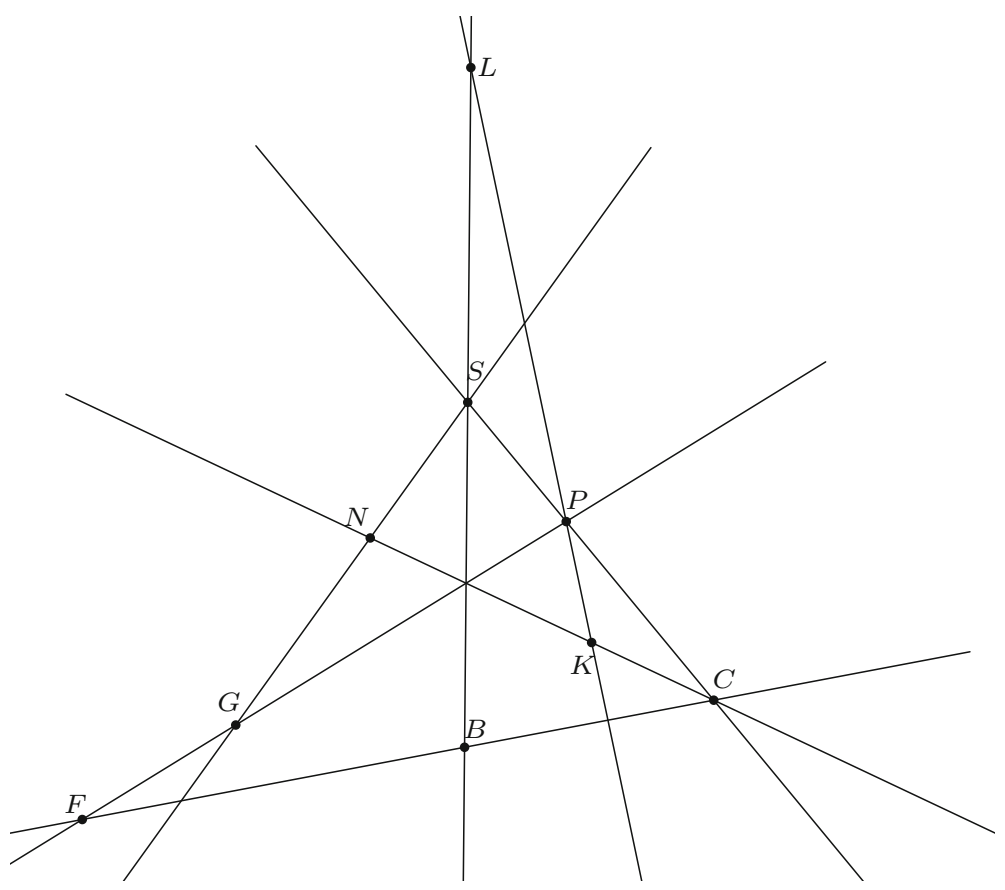

Fig. $5 \mathbb{B} 12$ construction - phase 4

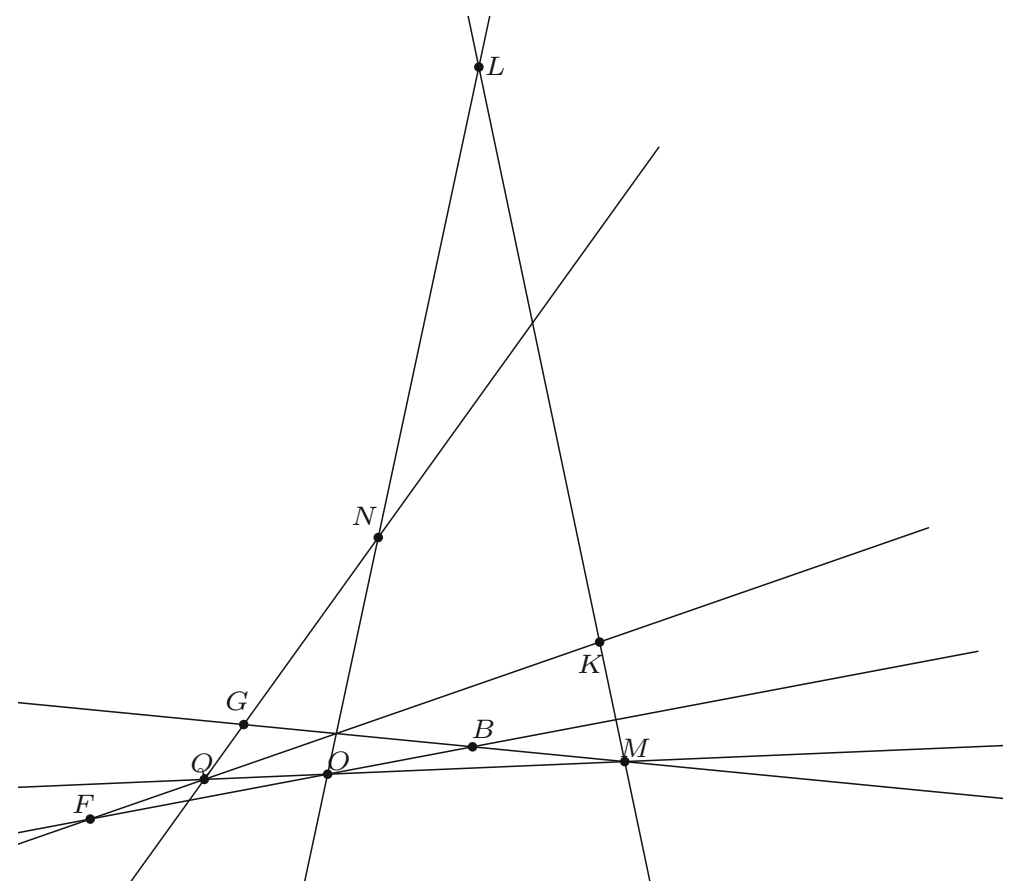

Fig. $6 \mathbb{B} 12$ construction - phase 5 


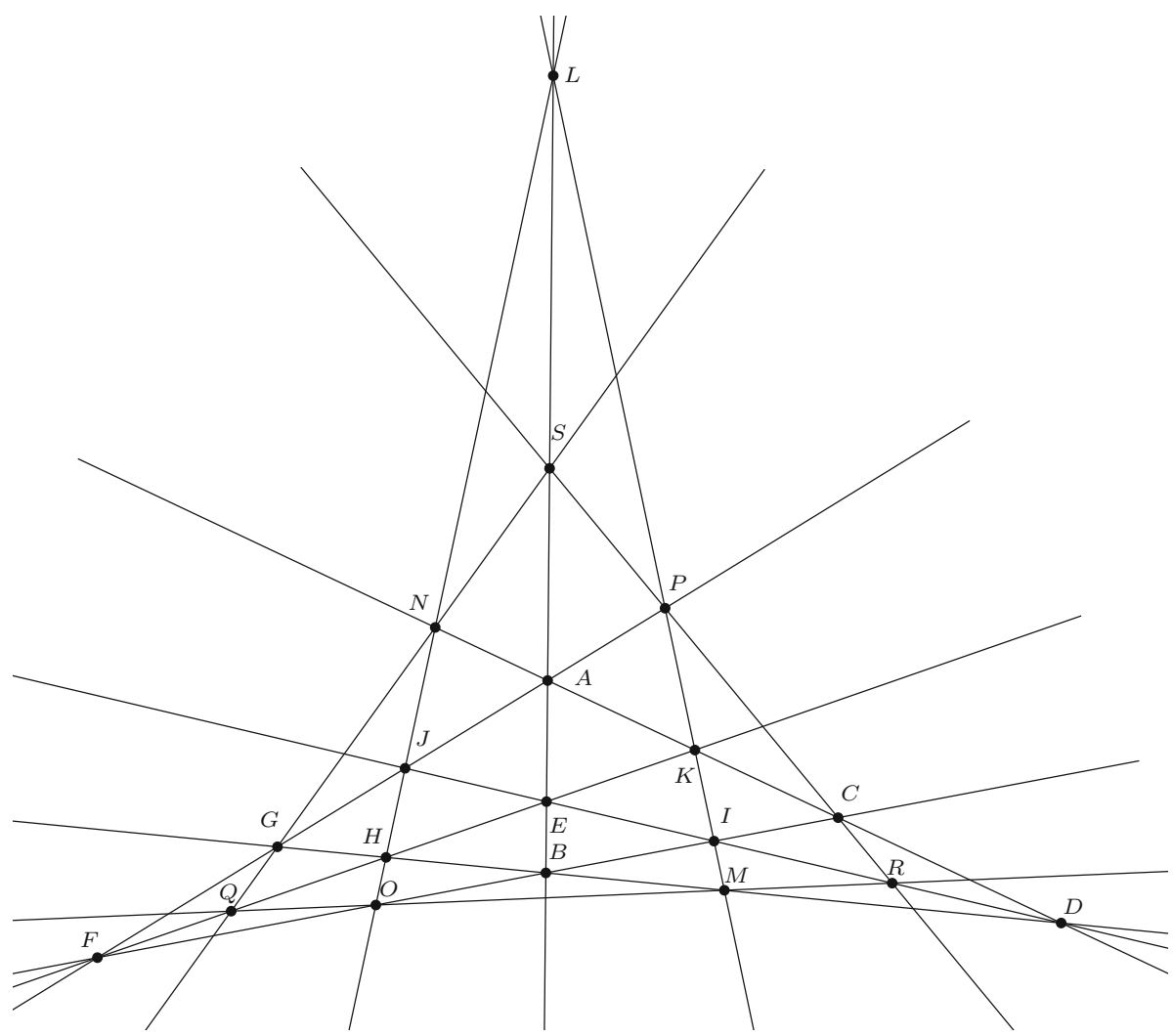

Fig. $7 \mathbb{B} 12$ - full configuration

$$
A=(1: 0: 0), \quad B=(0: 1: 0), \quad C=(0: 0: 1)
$$

Then we have the following equations of lines

$$
A B: z=0, \quad A C: y=0, \quad B C: x=0 .
$$

On these lines, we choose points $E, D, F$ respectively, all distinct from the fundamental points. Thus we may assume that their coordinates are:

$$
D=\left(a_{2}: 0: a_{1}\right), \quad E=\left(b_{1}: b_{2}: 0\right), \quad F=\left(0: c_{1}: c_{2}\right),
$$

with $a_{1}, a_{2}, b_{1}, b_{2}, c_{1}, c_{2} \neq 0$. Hence we obtain the following equations of lines

$$
\begin{aligned}
& A F: c_{2} y-c_{1} z=0, \\
& B D: a_{1} x-a_{2} z=0, \\
& E F: b_{2} c_{2} x-b_{1} c_{2} y+b_{1} c_{1} z=0, \\
& E D: a_{1} b_{2} x-a_{1} b_{1} y-a_{2} b_{2} z=0,
\end{aligned}
$$


which gives us the coordinates of points

$$
\begin{aligned}
G & =A F \cap B D=\left(a_{2} c_{2}: a_{1} c_{1}: a_{1} c_{2}\right), \\
H & =B D \cap E F=\left(a_{2} b_{1} c_{2}: a_{2} b_{2} c_{2}+a_{1} b_{1} c_{1}: a_{1} b_{1} c_{2}\right), \\
I & =B C \cap E D=\left(0: a_{2} b_{2}:-a_{1} b_{1}\right), \\
J & =A F \cap E D=\left(a_{1} b_{1} c_{1}+a_{2} b_{2} c_{2}: a_{1} b_{2} c_{1}: a_{1} b_{2} c_{2}\right), \\
K & =A C \cap E F=\left(b_{1} c_{1}: 0:-b_{2} c_{2}\right) .
\end{aligned}
$$

Then we obtain equations of the lines

$$
\begin{aligned}
& H J: a_{1} a_{2} b_{2}^{2} c_{2}^{2} x+a_{1}^{2} b_{1}^{2} c_{1} c_{2} y-\left(a_{1}^{2} b_{1}^{2} c_{1}^{2}+a_{2}^{2} b_{2}^{2} c_{2}^{2}+a_{1} a_{2} b_{1} b_{2} c_{1} c_{2}\right) z=0, \\
& I K: a_{2} b_{2}^{2} c_{2} x+a_{1} b_{1}^{2} c_{1} y+a_{2} b_{1} b_{2} c_{1} z=0,
\end{aligned}
$$

and the point $L=H J \cap I K=\left(a_{1} b_{1}^{2} c_{1}:-a_{2} b_{2}^{2} c_{2}: 0\right)$, hence it lies on the line through $A$ and $B$. This provides an alternative proof of Lemma 1 .

We find the coordinates of the remaining points

$$
\begin{aligned}
M & =B D \cap I K=\left(-a_{1} a_{2} b_{1}^{2} c_{1}: a_{2}^{2} b_{2}^{2} c_{2}+a_{1} a_{2} b_{1} b_{2} c_{1}:-a_{1}^{2} b_{1}^{2} c_{1}\right), \\
N & =A C \cap H J=\left(a_{1}^{2} b_{1}^{2} c_{1}^{2}+a_{2}^{2} b_{2}^{2} c_{2}^{2}+a_{1} a_{2} b_{1} b_{2} c_{1} c_{2}: 0: a_{1} a_{2} b_{2}^{2} c_{2}^{2}\right), \\
O & =H J \cap B C=\left(0: a_{1}^{2} b_{1}^{2} c_{1}^{2}+a_{2}^{2} b_{2}^{2} c_{2}^{2}+a_{1} a_{2} b_{1} b_{2} c_{1} c_{2}: a_{1}^{2} b_{1}^{2} c_{1} c_{2}\right), \\
P & =A F \cap I K=\left(a_{1} b_{1}^{2} c_{1}^{2}+a_{2} b_{1} b_{2} c_{1} c_{2}:-a_{2} b_{2}^{2} c_{1} c_{2}:-a_{2} b_{2}^{2} c_{2}^{2}\right) .
\end{aligned}
$$

The remaining lines are

$$
\begin{gathered}
N G: a_{1} a_{2} b_{2}^{2} c_{2}^{2} x+\left(a_{1}^{2} b_{1}^{2} c_{1} c_{2}+a_{1} a_{2} b_{1} b_{2} c_{2}^{2}\right) y \\
-\left(a_{1}^{2} b_{1}^{2} c_{1}^{2}+a_{2}^{2} b_{2}^{2} c_{2}^{2}+a_{1} a_{2} b_{1} b_{2} c_{1} c_{2}\right) z=0, \\
C P: a_{2} b_{2}^{2} c_{2} x+\left(a_{1} b_{1}^{2} c_{1}+a_{2} b_{1} b_{2} c_{2}\right) y=0,
\end{gathered}
$$

and finally we obtain the coordinates of points

$$
\begin{aligned}
Q= & E F \cap N G=\left(a_{2}^{2} b_{1} b_{2} c_{2}^{2}: a_{1}^{2} b_{1}^{2} c_{1}^{2}+2 a_{1} a_{2} b_{1} b_{2} c_{1} c_{2}+a_{2}^{2} b_{2}^{2} c_{2}^{2}: a_{1}^{2} b_{1}^{2} c_{1} c_{2}\right. \\
& \left.+2 a_{1} a_{2} b_{1} b_{2} c_{2}^{2}\right), \\
R= & D E \cap C P=\left(a_{1} a_{2} b_{1}^{2} c_{1}+a_{2}^{2} b_{1} b_{2} c_{2}:-a_{2}^{2} b_{2}^{2} c_{2}: a_{1}^{2} b_{1}^{2} c_{1}+2 a_{1} a_{2} b_{1} b_{2} c_{2}\right), \\
S= & C P \cap N G=\left(a_{1} b_{1}^{2} c_{1}+a_{2} b_{1} b_{2} c_{2}:-a_{2} b_{2}^{2} c_{2}: 0\right) .
\end{aligned}
$$

Again, it is now obvious that $S$ is collinear with $A$ and $B$. This gives an alternative proof of Lemma 2.

Checking the vanishing of determinants

$$
\left|\begin{array}{ccc}
-a_{2} a_{1} b_{1}^{2} c_{1} & a_{2}^{2} b_{2}^{2} c_{2}+a_{1} a_{2} b_{1} b_{2} c_{1} & -a_{1}^{2} b_{1}^{2} c_{1} \\
0 & a_{1}^{2} b_{1}^{2} c_{1}^{2}+a_{2}^{2} b_{2}^{2} c_{2}^{2}+a_{1} a_{2} b_{1} b_{2} c_{1} c_{2} & a_{1}^{2} b_{1}^{2} c_{1} c_{2} \\
a_{2}^{2} b_{1} b_{2} c_{2}^{2} & a_{1}^{2} b_{1}^{2} c_{1}^{2}+2 a_{1} a_{2} b_{1} b_{2} c_{1} c_{2}+a_{2}^{2} b_{2}^{2} c_{2}^{2} & a_{1}^{2} b_{1}^{2} c_{1} c_{2}+2 a_{2} a_{1} b_{1} b_{2} c_{2}^{2}
\end{array}\right|=0
$$

and

$$
\left|\begin{array}{ccc}
-a_{2} a_{1} b_{1}^{2} c_{1} & a_{2}^{2} b_{2}^{2} c_{2}+a_{1} a_{2} b_{1} b_{2} c_{1} & -a_{1}^{2} b_{1}^{2} c_{1} \\
0 & a_{1}^{2} b_{1}^{2} c_{1}^{2}+a_{2}^{2} b_{2}^{2} c_{2}^{2}+a_{1} a_{2} b_{1} b_{2} c_{1} c_{2} & a_{1}^{2} b_{1}^{2} c_{1} c_{2} \\
a_{1} a_{2} b_{1}^{2} c_{1}+a_{2}^{2} b_{1} b_{2} c_{2} & -a_{2}^{2} b_{2}^{2} c_{2} & a_{1}^{2} b_{1}^{2} c_{1}+2 a_{1} a_{2} b_{1} b_{2} c_{2}
\end{array}\right|=0
$$


we obtain the collinearity of points $M, O, Q$ and $M, O, R$, what finally implies that all points $M, O, Q, R$ lie on a common line and

$$
\begin{aligned}
& \text { MO: } a_{2}\left(2 a_{1}^{2} b_{2}^{2} c_{2}^{2}+a_{2}^{2} b_{1}^{2} c_{1}^{2}+2 a_{1} a_{2} b_{1} b_{2} c_{1} c_{2}\right) x+a_{1} a_{2}^{2} b_{1}^{2} c_{1} c_{2} y \\
& \quad-a_{1}\left(a_{2}^{2} b_{1}^{2} c_{1}^{2}+a_{1}^{2} b_{2}^{2} c_{2}^{2}+a_{1} a_{2} b_{1} b_{2} c_{1} c_{2}\right) z=0 .
\end{aligned}
$$

This gives an alternative proof of Lemma 3 .

Results presented in this section put together provide also a proof of Theorem A.

Proof of Theorem A The construction described above depends on parameters $\left(\left(a_{1}: a_{2}\right)\right.$, $\left.\left(b_{1}: b_{2}\right),\left(c 1: c_{2}\right)\right) \in\left(\mathbb{P}^{1}\right)^{3}$. In order to ensure that all 12 lines are distinct and also that their intersection points do not fall together, there is a number of relations between the parameters which must not be satisfied. All these relations are polynomial equations, thus they define proper closed subset in $\left(\mathbb{P}^{1}\right)^{3}$. They are explicitly discussed in the subsequent section. The complement of the degeneracy loci is a non-empty open set. Thus Theorem A is established.

\section{Parameter space for Böröczky configuration of 12 lines and degenerations}

All configurations in which the points $D, E, F$ are mutually distinct from the points $A, B, C$ are parameterized by 3 parameters in $\left(\mathbb{K}^{*}\right)^{3}$, where $\mathbb{K}$ is the ground field (either $\mathbb{R}$ or $\mathbb{Q}$ ). For various reasons it is convenient to work with a compact parameter space. A natural compactification coming here into the picture is $\mathcal{M}=\left(\mathbb{P}^{1}(\mathbb{K})\right)^{3}$. This is a multi-homogeneous space with coordinates $\left(a_{1}: a_{2}\right),\left(b_{1}: b_{2}\right)$ and $\left(c_{1}: c_{2}\right)$ introduced in Sect. 2.2. Our motivation to include degenerations stems also from the interest whether they lead to further containment counterexamples.

We evaluate now the conditions that all points and lines appearing in the construction are distinct. Comparing the coordinates of alls points and the equations of all lines we obtain the following degeneracy conditions:

(i) $a_{1} a_{2} b_{1} b_{2} c_{1} c_{2}=0$,

(ii) $a_{1} b_{1} c_{1}+a_{2} b_{2} c_{2}=0$,

(iii) $a_{1} b_{1} c_{1}+2 a_{2} b_{2} c_{2}=0$.

This means that if the numbers $a_{1}, \ldots, c_{2}$ do not satisfy any of above equations, then the points $A, \ldots, S$ defined in the previous section together with appropriate lines form a $\mathbb{B} 12$ configuration. The conditions (i), (ii) and (iii) define divisors $D_{1}, D_{2}, D_{3}$ in $\left(\mathbb{P}^{1}(\mathbb{K})\right)^{3}$ of multidegree $(2,2,2),(1,1,1)$, and $(1,1,1)$ respectively. Their union contains all degenerate configurations.

Below we present degenerations of our configuration corresponding to points in these divisors and their intersections.

\subsection{Degenerations coming from the divisor $D_{1}$}

We begin with the divisor $D_{1}$. This is the union of three pairs of disjoint "planes" $\left(\mathbb{P}^{1}(\mathbb{K}) \times\right.$ $\left.\mathbb{P}^{1}(\mathbb{K})\right)$ in $\mathcal{M}$. If the parameter $\mathfrak{m}=\left(\left(a_{1}: a_{2}\right),\left(b_{1}: b_{2}\right),\left(c_{1}: c_{2}\right)\right) \in \mathcal{M}$ is taken from $D_{1}$ but does not belong to its singular locus, then just one coordinate is zero. Without loss of generality we may assume that it is $a_{1}=0$, i.e. $D=A$. Then the configuration degenerates to the configuration of 7 lines with 6 triple points. These incidences are indicated in Fig. 8. 


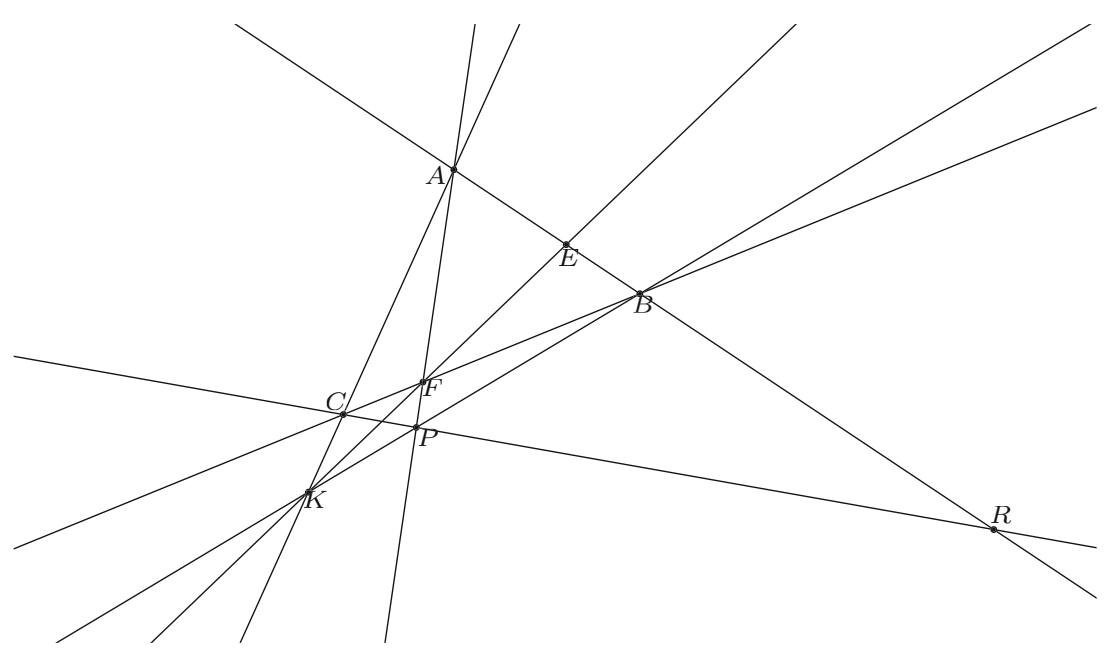

Fig. 8 Degeneration $a_{1}=0$

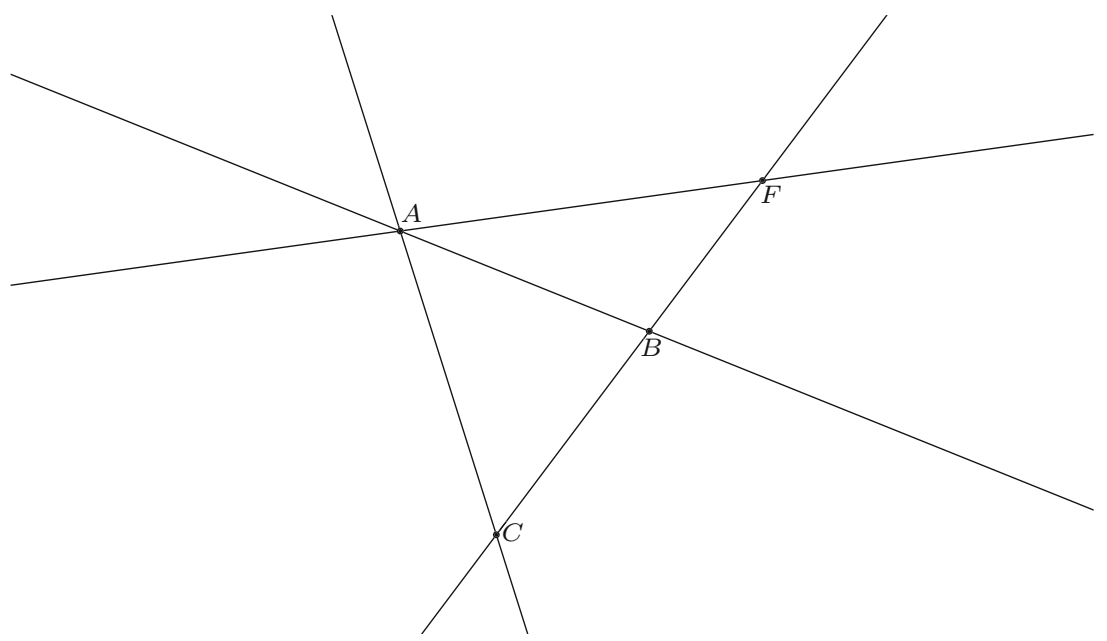

Fig. 9 Degeneration $a_{1}=b_{1}=0$

Let now $\mathfrak{m}$ be a double point on $D_{1}$. Without loss of generality, we may assume that it is defined by $a_{1}=b_{1}=0$. Then the whole configuration degenerates to a quasi-pencil on four lines, i.e. there are 4 lines and one triple point. The incidences are indicated in Fig. 9.

Finally, if $\mathfrak{m}$ is a triple point on $D_{1}$, then everything degenerates to the triangle with vertices $A, B, C$.

\subsection{Degenerations associated to $D_{2}$}

This case has an easy geometric interpretation, namely it occurs when the points $D, E, F$ are collinear, i.e. to begin with we get a Menelaus configuration. It implies that some points and lines in the generic configuration coincide. In fact there are only six lines left. They intersect in 4 triple and 3 double points. This is indicated in Fig. 10. 


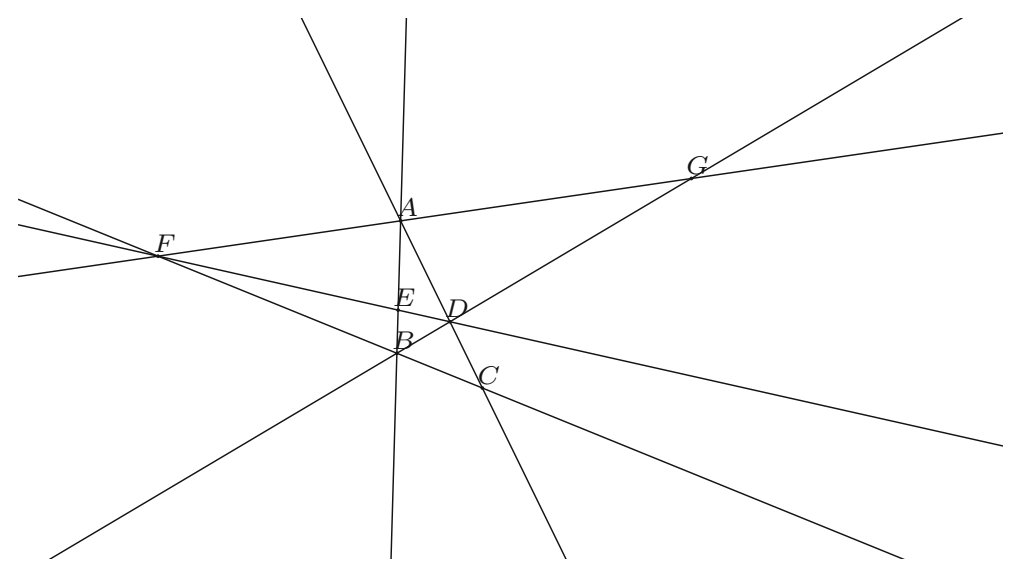

Fig. 10 Degeneration $D_{2}$

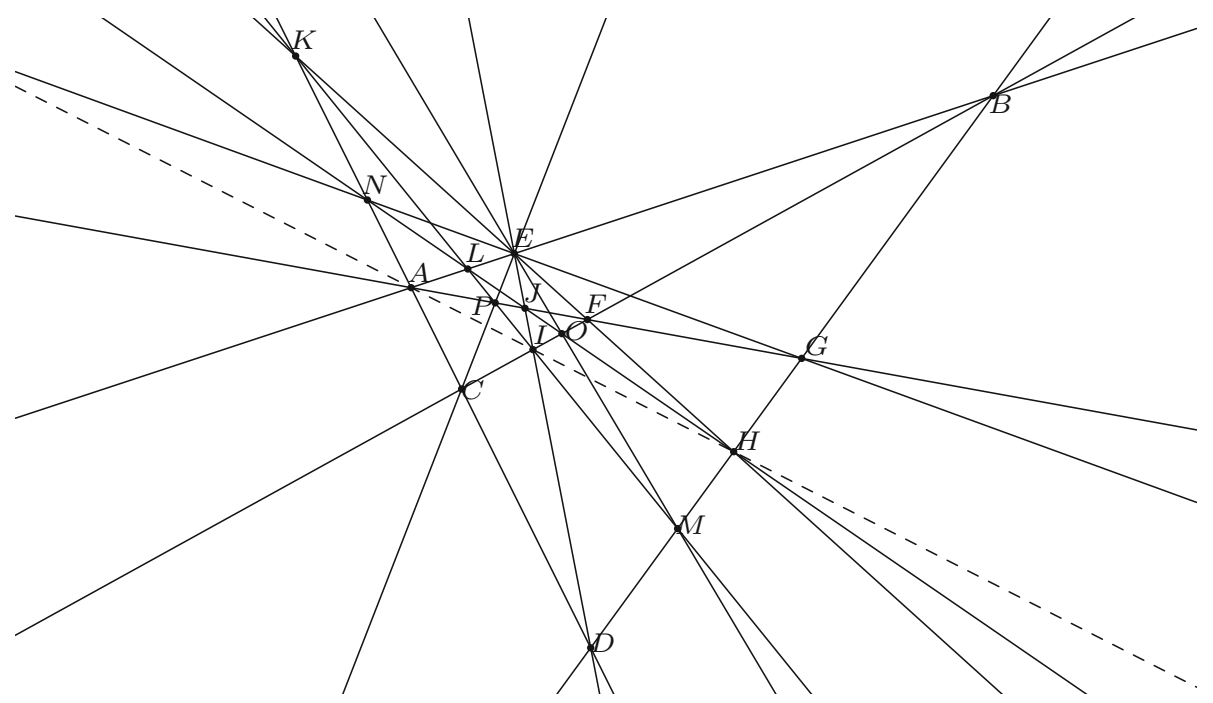

Fig. 11 Degeneration $D_{3}$

\subsection{Degenerations associated to divisor $D_{3}$}

The last degenerating condition is ( $\mathrm{iii}$ ). An interesting phenomenon in this case is that points $E, Q, R, S$ coincide so that the point $E$ turns to a sixtuple point, there are 15 triple points in this configuration and 6 double points, which are not named in Fig. 11 indicating the incidences in this case.

Note that, compared to the general case, there are additional collinearities of points: $N E G$, $C E P, E M O$ and $A I H$. The last mentioned line is not a line of the original configuration. We present incidences of this degeneration in Table 1.

The incidences between the divisors $D_{1}, D_{2}$ and $D_{3}$ are explained in the next remark.

Remark 2 The intersection locus $Z$ of any two divisors $D_{i}, D_{j}$ is contained also in the third divisor $D_{k}$, for $\{i, j, k\}=\{1,2,3\}$ and this set $Z$ is contained in the singular locus of divisor 
Table 1 Incidence table for degeneration (iii)

\begin{tabular}{|c|c|c|c|c|c|c|c|c|c|c|c|c|}
\hline & $\mathrm{AC}$ & $\mathrm{AB}$ & $\mathrm{BC}$ & $\mathrm{AF}$ & BD & $\mathrm{EF}$ & $\mathrm{DE}$ & HJ & IK & EG & $\mathrm{CE}$ & EM \\
\hline A & + & + & & + & & & & & & & & \\
\hline B & & + & + & & + & & & & & & & \\
\hline $\mathrm{C}$ & + & & + & & & & & & & & + & \\
\hline D & + & & & & + & & + & & & & & \\
\hline E & & + & & & & + & + & & & + & + & + \\
\hline $\mathrm{F}$ & & & + & + & & + & & & & & & \\
\hline G & & & & + & + & & & & & + & & \\
\hline $\mathrm{H}$ & & & & & + & + & & + & & & & \\
\hline I & & & + & & & & + & & + & & & \\
\hline $\mathrm{J}$ & & & & + & & & + & + & & & & \\
\hline $\mathrm{K}$ & + & & & & & + & & & + & & & \\
\hline $\mathrm{L}$ & & + & & & & & & + & + & & & \\
\hline $\mathrm{M}$ & & & & & + & & & & + & & & + \\
\hline $\mathrm{N}$ & + & & & & & & & + & & + & & \\
\hline $\mathrm{O}$ & & & + & & & & & + & & & & + \\
\hline $\mathrm{P}$ & & & & + & & & & & + & & + & \\
\hline
\end{tabular}

$D_{1}$. The degenerations corresponding to points in $Z$ (away of triple points of $D_{1}$ ) are indicated in Fig. 9.

We conclude the appendix by the following observation.

Corollary 2 None of degenerate configurations provides a counterexample to the containment problem. This shows in particular that being a counterexample configuration is not a closed condition.

Proof Since we have concrete coordinates to work with, the claim can be easily checked with a symbolic algebra program, we used Singular [7].

\section{A configuration of 15 lines with 31 triple points}

The configuration we are now interested in is visualized in Fig. 12. For clarity the points in the figure are labeled by numbers only. The number $i$ in the picture corresponds to the point $P_{i}$ in the text below.

\subsection{Construction}

In this section we construct a configuration of 15 lines with 31 triple points using the algebraic method. A geometric proof works along the same lines as in the case of a $\mathbb{B} 12$ configuration and we leave it to a motivated reader. To begin with let $P_{1}, P_{2}, P_{3}$ and $P_{4}$ be the standard points, i.e.

$$
P_{1}=(1: 0: 0), \quad P_{2}=(0: 1: 0), \quad P_{3}=(0: 0: 1), \quad P_{4}=(1: 1: 1)
$$




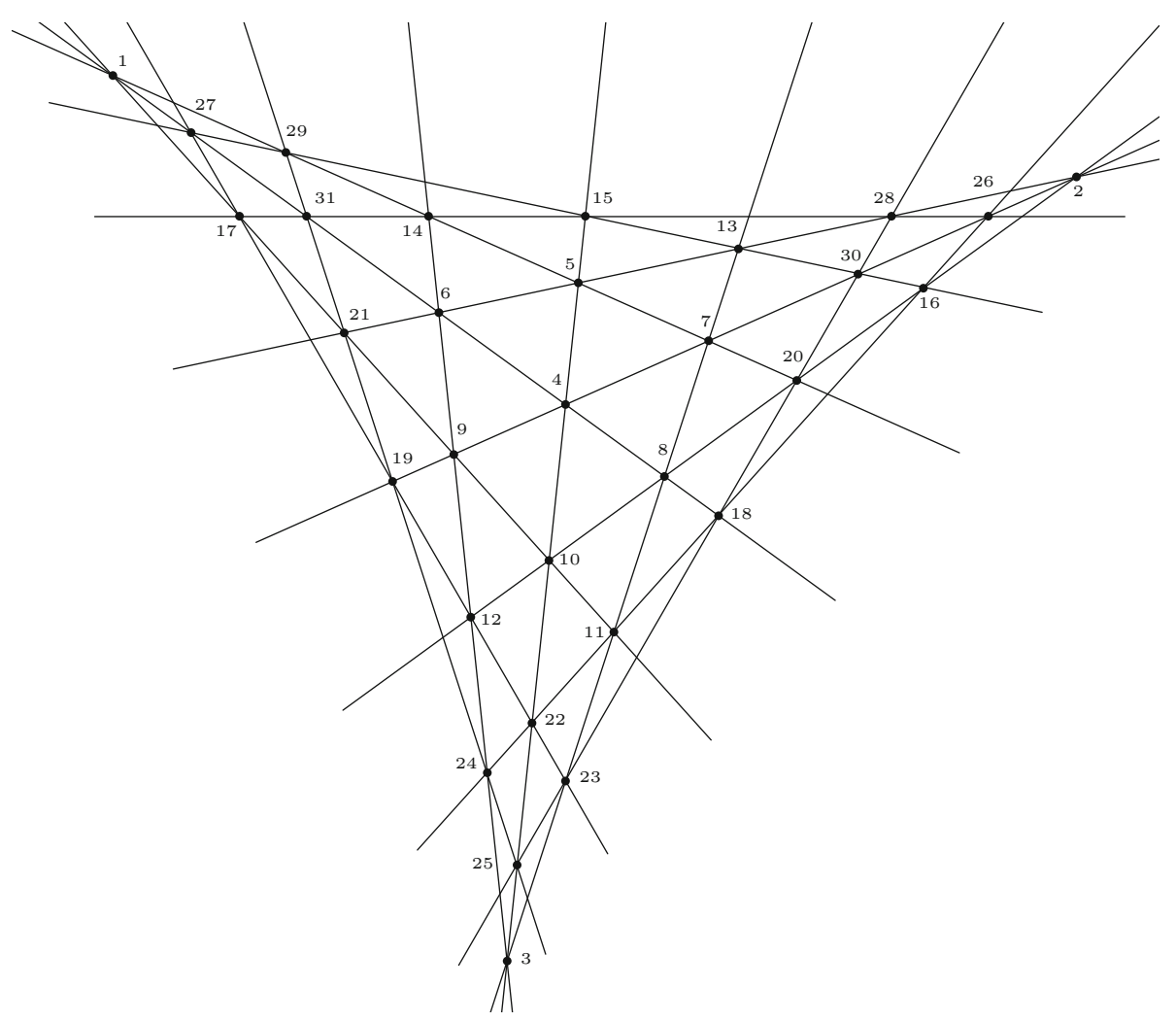

Fig. $12 \mathbb{B} 15$ configuration

Then we have the following equations of lines

$$
P_{1} P_{4}:-y+z=0, \quad P_{2} P_{4}: x-z=0, \quad P_{3} P_{4}:-x+y=0 .
$$

On the line $P_{3} P_{4}$ we choose a point $P_{5}$ distinct from the fundamental points. Thus we may assume that its coordinates are:

$$
P_{5}=(a: a: 1)
$$

with $a \notin\{0,1\}$. Hence we obtain the following equations of lines

$$
\begin{aligned}
& P_{1} P_{5}:-y+a z=0, \\
& P_{2} P_{5}: x-a z=0,
\end{aligned}
$$

which gives us coordinates of the points

$$
\begin{aligned}
& P_{6}=P_{1} P_{4} \cap P_{2} P_{5}=(a: 1: 1), \\
& P_{7}=P_{2} P_{4} \cap P_{1} P_{5}=(1: a: 1) .
\end{aligned}
$$

Then we obtain the following equations of the lines and coordinates of points

$$
\begin{gathered}
P_{3} P_{6}:-x+a y=0, \\
P_{3} P_{7}: a x-y=0,
\end{gathered}
$$




$$
\begin{aligned}
P_{8}= & P_{1} P_{4} \cap P_{3} P_{7}=(1: a: a), \\
& P_{2} P_{8}: a x-z=0, \\
P_{9}= & P_{2} P_{4} \cap P_{3} P_{6}=(a: 1: a), \\
& P_{1} P_{9}:-a y+z=0, \\
P_{10}= & P_{2} P_{8} \cap P_{3} P_{4}=(1: 1: a) .
\end{aligned}
$$

Fact 1 The incidence $P_{10} \in P_{1} P_{9}$ follows from above choices.

Further we obtain coordinates of the points

$$
\begin{aligned}
& P_{11}=P_{3} P_{7} \cap P_{1} P_{9}=\left(1: a: a^{2}\right), \\
& P_{12}=P_{3} P_{6} \cap P_{2} P_{8}=\left(a: 1: a^{2}\right), \\
& P_{13}=P_{2} P_{5} \cap P_{3} P_{7}=\left(a: a^{2}: 1\right), \\
& P_{14}=P_{1} P_{5} \cap P_{3} P_{6}=\left(a^{2}: a: 1\right) .
\end{aligned}
$$

Now we encounter the second choice in this construction. On the line $P_{3} P_{4}$ we choose a point $P_{15}$ distinct from the fundamental points and the point $P_{5}$. Thus we may assume that its coordinates are:

$$
P_{15}=(b: b: 1)
$$

with $b \notin\{0,1, a\}$.

Then we obtain the following equations of lines and coordinates of points

$$
\begin{aligned}
& P_{14} P_{15}:(-a+b) x+\left(a^{2}-b\right) y+\left(-a^{2} b+a b\right) z=0, \\
& P_{13} P_{15}:\left(-a^{2}+b\right) x+(a-b) y+\left(a^{2} b-a b\right) z=0, \\
& P_{16}=P_{2} P_{8} \cap P_{13} P_{15}=\left(a-b:-a^{3} b+a^{2} b+a^{2}-b: a^{2}-a b\right), \\
& P_{17}=P_{1} P_{9} \cap P_{14} P_{15}=\left(a^{3} b-a^{2} b-a^{2}+b:-a+b:-a^{2}+a b\right), \\
& P_{11} P_{16}:\left(-a^{5} b+a^{4} b+a^{4}-a^{3}\right) x+\left(-a^{3}+a^{2} b+a^{2}-a b\right) y \\
& \quad+\left(a^{3} b-a^{2} b-a b+b\right) z=0, \\
& P_{12} P_{17}:\left(-a^{3}+a^{2} b+a^{2}-a b\right) x+\left(-a^{5} b+a^{4} b+a^{4}-a^{3}\right) y \\
& \quad+\left(a^{3} b-a^{2} b-a b+b\right) z=0, \\
& P_{18}=P_{1} P_{4} \cap P_{11} P_{16} \\
& \quad=\left(-a^{3} b+a^{3}-a^{2}+2 a b-b:-a^{5} b+a^{4} b+a^{4}-a^{3}:-a^{5} b+a^{4} b+a^{4}-a^{3}\right), \\
& P_{19}=P_{2} P_{4} \cap P_{12} P_{17} \\
& \quad=\left(-a^{5} b+a^{4} b+a^{4}-a^{3}:-a^{3} b+a^{3}-a^{2}+2 a b-b:-a^{5} b+a^{4} b+a^{4}-a^{3}\right), \\
& P_{20}=P_{1} P_{5} \cap P_{2} P_{8}=\left(1: a^{2}: a\right), \\
& P_{21}=P_{2} P_{5} \cap P_{1} P_{9}=\left(a^{2}: 1: a\right), \\
& P_{18} P_{20}:\left(a^{7} b-2 a^{6} b-a^{6}+a^{5} b-a^{4}\right) x+\left(-a^{5} b+2 a^{4} b-2 a^{2} b+a b\right) y \\
& +\left(a^{5}-a^{4} b-2 a^{4}+2 a^{3} b+a^{3}-a^{2} b\right) z=0,
\end{aligned}
$$




$$
\begin{aligned}
& P_{19} P_{21}:\left(-a^{5} b+2 a^{4} b-2 a^{2} b+a b\right) x+\left(a^{7} b-2 a^{6} b-a^{6}+a^{5} b-a^{4}\right) y \\
& \quad+\left(a^{5}-a^{4} b-2 a^{4}+2 a^{3} b+a^{3}-a^{2} b\right) z=0, \\
& P_{22}=P_{3} P_{4} \cap P_{11} P_{16} \\
& \quad=\left(a^{3} b-a^{2} b-a b+b: a^{3} b-a^{2} b-a b+b: a^{5} b-a^{4} b-a^{4}+2 a^{3}-a^{2} b-a^{2}+a b\right) .
\end{aligned}
$$

Fact 2 Note that the incidence $P_{22} \in P_{12} P_{17}$ does not impose any additional conditions on $a$ and $b$.

The coordinates of the remaining points are now easy to find:

$$
\begin{aligned}
& P_{23}=P_{3} P_{7} \cap P_{12} P_{17}=\left(-a^{3} b+a^{2} b+a b-b:-a^{4} b+a^{3} b+a^{2} b-a b:\right. \\
& \left.-a^{6} b+a^{5} b+a^{5}-a^{4}-a^{3}+a^{2} b+a^{2}-a b\right), \\
& P_{24}=P_{3} P_{6} \cap P_{11} P_{16}=\left(-a^{4} b+a^{3} b+a^{2} b-a b:-a^{3} b+a^{2} b+a b-b:\right. \\
& \left.-a^{6} b+a^{5} b+a^{5}-a^{4}-a^{3}+a^{2} b+a^{2}-a b\right), \\
& P_{25}=P_{3} P_{4} \cap P_{18} P_{20}=\left(a^{5}-a^{4} b-2 a^{4}+2 a^{3} b+a^{3}-a^{2} b:\right. \\
& a^{5}-a^{4} b-2 a^{4}+2 a^{3} b+a^{3}-a^{2} b \text { : } \\
& \left.-a^{7} b+2 a^{6} b+a^{6}-2 a^{5}-2 a^{4} b+a^{4}+2 a^{2} b-a b\right), \\
& P_{26}=P_{2} P_{4} \cap P_{11} P_{16}=\left(-a^{3}+a^{2} b+a^{2}-a b:\right. \\
& a^{5} b-a^{4} b-a^{4}-a^{3} b+a^{3}+a^{2} b+a b-b: \\
& \left.-a^{3}+a^{2} b+a^{2}-a b\right) \\
& P_{27}=P_{1} P_{4} \cap P_{12} P_{17}=\left(a^{5} b-a^{4} b-a^{4}-a^{3} b+a^{3}+a^{2} b+a b-b\right. \text { : } \\
& \left.-a^{3}+a^{2} b+a^{2}-a b:-a^{3}+a^{2} b+a^{2}-a b\right), \\
& P_{28}=P_{2} P_{5} \cap P_{14} P_{15}=\left(a^{3}-a b: a^{2} b+a^{2}-2 a b: a^{2}-b\right), \\
& P_{29}=P_{1} P_{5} \cap P_{13} P_{15}=\left(a^{2} b+a^{2}-2 a b: a^{3}-a b: a^{2}-b\right), \\
& P_{30}=P_{2} P_{4} \cap P_{13} P_{15}=\left(a-b:-a^{2} b+a^{2}+a b-b: a-b\right), \\
& P_{31}=P_{1} P_{4} \cap P_{14} P_{15}=\left(-a^{2} b+a^{2}+a b-b: a-b: a-b\right) .
\end{aligned}
$$

Fact 3 It is easy to see that $P_{25} \in P_{19} P_{21}$.

Finally we have to check under which conditions the following incidences are satisfied

$$
\begin{aligned}
& P_{23} \in P_{18} P_{20}, \quad P_{24} \in P_{19} P_{21}, \quad P_{26} \in P_{14} P_{15}, \quad P_{27} \in P_{13} P_{15}, \\
& P_{28} \in P_{18} P_{20}, \quad P_{29} \in P_{19} P_{21}, \quad P_{30} \in P_{18} P_{20}, \quad P_{31} \in P_{19} P_{21} \text {. }
\end{aligned}
$$

Evaluating algebraic conditions we obtain polynomial equations involving $a$ and $b$ and the smallest ideal that contains all these polynomials is the ideal generated by the polynomial

$$
(a-1)^{2}\left(a^{4} b-a^{2} b^{2}-a^{3}+a^{2} b-a b^{2}+b^{2}\right) .
$$


Since by assumption $a \neq 1$, it must be

$$
f:=a^{4} b-a^{2} b^{2}-a^{3}+a^{2} b-a b^{2}+b^{2}=0 .
$$

\subsection{The parameter curve}

The polynomial $f$ in (13) defines a singular curve in the plane $\mathbb{R}^{2}$ with coordinates $(a, b)$. We want to pass to its smooth model. More precisely, we are interested in the minimal Weierstrass form of this curve, see [15, Chapter III, § 3]. To this end we substitute

$$
b=\frac{(a-1) a T+a^{2}+a^{4}}{2\left(a^{2}+a-1\right)}
$$

into the Eq. (13) and we get

$$
(a-1)^{2} a^{2}\left(-a^{4}-2 a^{3}-5 a^{2}+T^{2}-4 a\right)=0 .
$$

We can again localize at $a=1$, so that it is enough to study the curve

$$
C: T^{2}=a(1+a)\left(4+a+a^{2}\right) .
$$

Performing additional substitutions

$$
a=\frac{1}{X}, \quad T=\frac{2 Y+X+1}{X^{2}}
$$

we obtain a smooth elliptic curve $E$ in the canonical form

$$
E: Y^{2}+X Y+Y=X^{3}+X^{2} .
$$

\begin{tabular}{|c|c|c|c|c|c|c|}
\hline Type & Parameters & Number of lines & $t_{2}$ & $t_{3}$ & $t_{4}$ & $t_{5}$ \\
\hline I & $a=b=1$ & 3 & 0 & 1 & 0 & 0 \\
\hline II & $\begin{array}{l}a=b=0 \\
a=b=-1\end{array}$ & 6 & 3 & 4 & 0 & 0 \\
\hline III & $\begin{array}{l}a=-\frac{1}{2} \pm \frac{\sqrt{3}}{2} i \\
b=-\frac{1}{2}\end{array}$ & 10 & 3 & 10 & 2 & 0 \\
\hline IV & $\begin{array}{l}a=-\frac{1}{2} \pm \frac{\sqrt{3}}{2} i \\
\mathrm{~b}=1\end{array}$ & 11 & 6 & 9 & 2 & 1 \\
\hline V & $\begin{array}{l}a=-\frac{1}{2} \pm \frac{1}{2} \sqrt{5-2 \sqrt{5}} i \\
b=\frac{1-\sqrt{5}}{4} \mp \frac{\sqrt{250-110 \sqrt{5}}}{20} i \\
a=-\frac{1}{2} \mp \frac{1}{2} \sqrt{5+2 \sqrt{5}} i \\
b=\frac{1+\sqrt{5}}{4} \mp \frac{\sqrt{250+110 \sqrt{5}}}{20} i\end{array}$ & 15 & 0 & 25 & 0 & 3 \\
\hline
\end{tabular}

This is the parameter space for $\mathbb{B} 15$ configurations and thus Theorem $\mathrm{B}$ is proved.

It is known (see Cremona basis [4]) that $E$ contains only 4 rational points. Each of them corresponds to forbidden values of $a$ and $b$. Thus Corollary 1 follows.

Table 2 Degenerations of the $\mathbb{B} 15$ configuration 


\section{Degenerations of $\mathbb{B} 15$ configurations}

Proceeding as in Sect. 3, i.e., evaluating the conditions for all points in the $\mathbb{B} 15$ configuration to be distinct, we obtain the following list of degenerated configurations (we omit tedious computations). The numbers $t_{k}$ in Table 2 denote the number of points where exactly $k$ lines meet.

There is one additional degeneration point at infinity, which corresponds to a degeneration of type II.

Remark 3 Degeneration of type $\mathrm{V}$ is exactly the Fermat configuration of 15 lines. It provides a counterexample to the containment in (1), see [14] for details.

Final remark Some preliminary calculations suggest that parameter spaces for $\mathbb{B} 3 k$ configurations with $k \geq 6$ are curves of genus $\geq 2$. We hope to come back to their study in the near future.

Acknowledgements We would like to thank Maciej Ulas for helping us with arithmetic questions. We thank also Tomasz Szemberg for valuable conversations. Finally we are grateful to the referee for reading our manuscript carefully and providing detailed interesting and stimulating remarks and suggestions.

Open Access This article is distributed under the terms of the Creative Commons Attribution 4.0 International License (http://creativecommons.org/licenses/by/4.0/), which permits unrestricted use, distribution, and reproduction in any medium, provided you give appropriate credit to the original author(s) and the source, provide a link to the Creative Commons license, and indicate if changes were made.

\section{References}

1. Baralić, D., Spasojević, I.: Illumination of Pascals Hexagrammum and Octagrammum Mysticum. Discrete Comput. Geom. 53, 414-427 (2015)

2. Bauer, T., Di Rocco, S., Harbourne, B., Huizenga, J., Lundman, A., Pokora, P., Szemberg, T.: Bounded negativity and arrangements of lines. Int. Math. Res. Not. 2015, 9456-9471 (2015)

3. Bauer, T., Harbourne, B., Knutsen, A.L., Küronya, A., Müller-Stach, S., Roulleau, X., Szemberg, T.: Negative curves on algebraic surfaces. Duke Math. J. 162, 1877-1894 (2013)

4. Cremona, J.E.: Elliptic curve data. http://johncremona.github.io/ecdata/

5. Crowe, D.W., McKee, T.A.: Sylvester's problem on collinear points. Math. Mag. 41(1), 30-34 (1968)

6. Czapliński, A., Główka-Habura, A., Malara, G., Lampa-Baczyńska, M., Łuszcz-Świdecka, P., Pokora, P., Szpond, J.: A counterexample to the containment $I^{(3)} \subset I^{2}$ over the reals. Adv. Geom. 16, 77-82 (2016)

7. Decker, W., Greuel, G.-M., Pfister, G., Schönemann, H.: Singular 4-0-2 — A computer algebra system for polynomial computations. http://www.singular.uni-kl.de(2015)

8. Dumnicki, M., Harbourne, B., Nagel, U., Seceleanu, A., Szemberg, T., Tutaj-Gasińska, H.: Resurgences for ideals of special point configurations in $\mathbb{P}^{N}$ coming from hyperplane arrangements. J. Algebra 443, 383-394 (2015)

9. Dumnicki, M., Harrer, D., Szpond, J.: On absolute linear Harbourne constants. preprint (2015). arXiv: 1507.04080

10. Dumnicki, M., Szemberg, T., Tutaj-Gasińska, H.: Counterexamples to the $I^{(3)} \subset I^{2}$ containment. J. Algebra 393, 24-29 (2013)

11. Füredi, Z., Palasti, I.: Arrangments of lines with a large number of triangles. Proc. Am. Math. Soc. 92, 561-566 (1984)

12. Green, B., Tao, T.: On sets defining few ordinary lines. Discrete Comput. Geom. 50, 409-468 (2013)

13. Harbourne, B., Seceleanu, A.: Containment counterexamples for ideals of various configurations of points in $\mathbb{P}^{N}$. J. Pure Appl. Algebra 219, 1062-1072 (2015)

14. Seceleanu, A.: A homological criterion for the containment between symbolic and ordinary powers of some ideals of points in $\mathbb{P}^{2}$. J. Pure Appl. Alg. 219, 4857-4871 (2015) 
15. Silverman, J.H.: The arithmetic of elliptic curves, 2nd edn. In: Graduate Texts in Mathematics, vol 106. Springer, Dordrecht (2009)

16. Sturmfels, B.: Computational algebraic geometry of projective configurations. J. Symbolic Comput. 11, 595-618 (1991)

17. Szemberg, T., Szpond, J.: On the containment problem. Rend. Circ. Mat. Palermo II. Ser. (2016). doi:10. 1007/s12215-016-0281-7

18. Szpond, J.: On linear Harbourne constants. Br. J. Math. Comput. Sci. 8, 286-297 (2015)

19. Tao, T.: Algebraic combinatorial geometry: the polynomial method in arithmetic combinatorics, incidence combinatorics, and number theory. EMS Surv. Math. Sci. 1, 1-46 (2014) 